\title{
De la campagne à la ville à la recherche de protection : Le cas colombien du déplacement forcé
}

\author{
Flor Edilma Osorio Pérez
}

\section{Résumé}

Le présent article traite de quelques dynamiques du déplacement forcé interne, présent de façon importante en Colombie pendant la dernière décennie à cause de la guerre interne que connaît ce pays. Suivant le flux ruralurbain, les paysans fuient leur domicile pour aller vers la ville. L'imaginaire de sécurité des villes, comme par exemple Bogotá où sont présents le gouvernement et toutes les institutions publiques, est en train de produire une urbanisation accélérée face à une déruralisation qui transforme l'ensemble du pays. La rencontre avec d'autres déplacés dans les mêmes circonstances que soi permet de tisser de nouvelles sociabilités improvisées, fragmentées et ambiguës et rend possible de trouver quelques réponses à leur situation. Malgré une précarité quotidienne, les desplazados (déplacés) soutiennent l'attente d'un futur meilleur, à défaut d'autres horizons et au sein d'une lassitude provoquée par l'instabilité et l'incertitude.

\section{Abstract \\ This article discusses some dynamics of internal forced dis- placement, which has occurred at a high level in Colum- bia over the last decade as a result of the civil war which the country is going through. Influenced by the rural-urban population flow, peasants flee from their homes to the imagined safety and security of the cities. Bogotá, for ex- ample, the seat of government and of all public institu- tions, is experiencing rapid urbanization, as distinct from a deruralization process which is transforming the whole country. When displaced people are able to meet with oth- ers in similar circumstances they are able to establish new improvised ties of sociability, fragmented and ambiguous as they are, and enables them to find some solutions to}

their circumstances. Despite the precariousness that each day brings, the desplazados (displaced) live in expectation of a better future, despite having no other place to go and being surrounded by a weariness caused by instability and uncertainty.

J'ai vu la fumée et les flammes monter jusqu'au ciel. «Mi gente » fuir de leurs maisons. Cris, horreur, pleurs et deuil. La mort est en liberté. Ta vie est en jeu, ton sang est le pari. Ils viennent avec des armes à feu, la mort est en liberté. Colombia est en jeu. Dans cette guerre maudite, autant de pauvres autant de morts. Je veux retourner au bord de ma rivière, je veux retourner à mon village. Aidez-nous mon Dieu à éteindre cet enfer ${ }^{1}$.

$\mathrm{E}$ n Colombie, près de quatre millions de personnes ont du fuir leur domicile pour échapper aux groupes armés et à la violence d'une guerre de plus en plus présente et complexe ${ }^{2}$ qui laisse sur son passage mort, désolation, déracinement et douleur. Les expulsions, déplacement forcé de la population, sont devenues une expression quotidienne dramatiquement banale de la violence armée. Elles marquent une rupture brutale et radicale avec la communauté et le territoire d'origine; elles traduisent une privation violente des repères matériels et symboliques qui fondent l'identité des individus et des familles qui en sont victimes. Pour les desplazados (déplacés), écartelés entre un présent amer, déjà inscrit dans le passé, et un futur incertain, le désespoir est absolu. Alors, où chercher protection? Les villes et centres peuplés, surtout les plus importants, sont les élus. L'imaginaire de sécurité dans les villes, où sont présents le gouvernement et toutes les institutions publiques, est en train de produire une urbanisation accélérée face à une déruralisation qui transforme l'ensemble du pays.

Ces réflexions suivent une partie de ma thèse doctorale qui a étudiée la situation générale du déplacement en Colombie, en accordant une importance particulière à la rup- 
ture et la reconstruction du territoire, des référents identitaires et de l'action collective ${ }^{3}$. Le texte se divise en quatre parties. Nous montrons d'abord le processus de déruralisation et d'urbanisation. Nous exposons ensuite quelques dynamiques du déplacement orienté vers Bogotá, la ville capitale, d'après les témoignages des déplacés. Nous abordons ensuite la catégorie desplazado qui tend à devenir substantielle et stigmatisée. Enfin, nous nous penchons sur les expressions de solidarité, d'organisation et de résistance des déplacés dans les villes.

\section{Processus accélérés de déruralisation et d'urbanisation}

En Colombie les campagnes ont occupé dans la guerre un espace privilégié, bien que non exclusif, pendant les cinquante dernières années au moins, espace qui garde une forte relation avec la subordination et l'exclusion des campagnes. C'est pourquoi le déplacement forcé concerne essentiellement les habitants ruraux. Guerre et déplacement sont en train de produire une déruralisation dont la contrepartie est une urbanisation accélérée, avec en arrière-plan une modernisation apparente, fragmentée et appauvrissante.

Le développement de la guerre dans les territoires ruraux obéit aux intérêts militaires, sociopolitiques et économiques des différents acteurs armés alliés aux acteurs non armés. Dans cette guerre complexe qu'est en train de vivre le pays le rapport de cause à effet devient diffus, à tel point qu'il est difficile d'établir d'une manière générale s'il y a des desplazados parce qu'il y a une guerre, ou s'il y a une guerre pour qu'il y ait des desplazados ${ }^{4}$. Au milieu des dynamiques régionales, la guerre généralisée recrée peu à peu ses particularités. Cependant, qu'environ $70 \%$ des foyers déplacés ont un rapport à la terre (à travers la résidence, l'emploi et/ou la propriété de la terre) demeure significatif. Avec le déplacement forcé des familles, près de quatre millions d'hectares se sont vus « désertés » et réinvestis. Le déplacement sert ainsi un double objectif : homogénéiser la population pour faciliter le contrôle du territoire et, en même temps, réordonner la propriété de la terre en fonction des intérêts des groupes armés.

La guerre en Colombie est en train de provoquer d'une manière accélérée et arbitraire une transformation du système social et économique de la campagne et des villes. Près de mille paysans entrent chaque jour dans un processus de transit forcé vers des centres urbains sans possibilité de retour, tout au moins à moyen terme. Au milieu d'une espèce de nomadisme temporaire, les foyers et les personnes en déplacement s'orientent vers les centres urbains qui voient s'accélérer ces dynamiques d'urbanisation. Plongés dans l'ambiguïté constante, les desplazados vivent dans la rupture et l'articulation forcée de deux logiques qui semblent s'inscrire de manière schématique entre tradition et modernité ${ }^{5}$, que l'on peut comparer avec la dichotomie campagne-ville. Cette polarisation théorique est cependant erronée. Dans la réalité, elle entretient constamment des articulations, des superpositions et des frontières diffuses.

Se déplacer suppose un changement forcé du territoire, qui rend obligatoire la construction de relations avec d'autres lieux, d'autres acteurs et d'autres groupes sociaux. Partir, sous la menace des armes, entraîne aussi une modification radicale de l'image que l'on a de soi-même et donc la construction d'une identité nouvelle. La guerre bouleverse donc les référents territoriaux de la population et, par-là, elle touche directement les dynamiques de construction identitaire. La terreur, les persécutions, les menaces modifient les représentations de l'espace et des objets ainsi que de la place qu'y occupent les individus et les groupes sociaux.

L'itinéraire campagne-ville paraît être le plus fréquent dans la dynamique du desplazamiento (déplacement) forcé ${ }^{6}$. Dans le cas colombien, les paysans partent en direction des centres urbains, qui ne sont pas uniquement les grandes villes mais n'importe quel périmètre urbain voisin, à la recherche de protection. Cependant, les centres urbains ne constituent pas automatiquement des espaces de plus grande sécurité physique par rapport à l'isolement et au manque de protection des campagnes. Dans certains cas, la garantie qu'ils offrent n'est pas plus grande. Voyons quelques manifestations de cette relation ambiguë du milieu urbain comme lieu sûr et comme alternative à la guerre :

1. Les centres urbains, même petits, confèrent un certain pouvoir d'action collective face aux demandes d'aide, de sorte que les distances et la solitude des campagnes restreignent la possibilité de donner et de recevoir de l'aide en cas d'agression. Cependant, rien n'est moins sûr, du fait que les actions violentes dans les petites municipalités se sont multipliées sans que leurs habitants aient pu y faire quoi que ce soit ${ }^{7}$. En même temps, dans les centres peuplés, il peut être plus facile d'établir des accords avec les acteurs armés de façon à éviter des irruptions violentes au sein des populations et des communautés ${ }^{8}$.

2. Les centres urbains peuvent garantir la défense de leurs citoyens grâce à la présence d'autorités telles les forces de l'ordre, habituellement présentes pour assurer cette tâche. Or, ceci est un imaginaire incertain, car les «prises de villages» sont fréquentes de la part des guérillas et des paramilitaires, sans qu'une plus grande protection soit offerte ${ }^{9}$. D'autre part, la présence de paramilitaires et de la guérilla dans le contrôle politique et militaire des populations rurales paraît indiquer 
qu'un autre élément empêche l'exercice de la protection de la population ${ }^{10}$. Même dans les villes de moyenne et de grande taille, les persécutions sélectives perpétrées sur des leaders et sur des personnes se maintiennent.

3. Les villes sont des lieux où peuvent se produire des rapports de force avec le gouvernement. Dans certains cas, on a vu des desplazamientos massifs dont le but était de faire pression sur le gouvernement et sur l'opinion publique, justement pourquoi ils s'orientent vers des villes stratégiques au niveau de l'économie et de la sécurité de la région ${ }^{11}$. Cependant, ce mécanisme a souvent échoué dans la mesure où à la suite de grands efforts et de privations de la population qui se déplace, ainsi que de la part de celle qui, par la force, héberge la première, l'exercice du retour des desplazados constitue un jeu de promesses non tenues. Ceci a pour effet de produire lassitude et incrédulité au sein de la population paysanne qui ne voit ni les résultats, ni les actions institutionnelles tant de développement socio-économique que de sécurité et de protection de leurs personnes.

La valorisation des espaces qui offrent ou non protection est en étroite relation avec la polarité campagne-ville, bien que ce soit seulement l'imaginaire de la population. L'occupation et le contrôle de territoires par les groupes armés, bien que couvrant les centres urbains stratégiques, a son siège privilégié dans les zones rurales et d'accès difficile ${ }^{12}$. Le flux rural-urbain qui suit le desplazamiento forcé interne reproduit en bonne partie les circuits migratoires traditionnels. Le rôle de la guerre dans les années 90 comme accélérateur de l'urbanisation ${ }^{13}$ dans l'ensemble du pays ne peut pas être valorisé avec suffisamment de précision. Cependant, à partir de perspectives locales, ces processus paraissent se confirmer.

Le milieu rural est synonyme d'espace dangereux, de sorte que la ville est identifiée comme un espace de protection et d'aide. Ceci renforce un processus d'urbanisation galopante, au milieu d'une dévalorisation économique, sociale et politique des campagnes qui se concrétise en politiques de découragement envers l'économie paysanne et agricole, insoutenable en conditions de conflit armé. Par cette voie, les guerres intestines imposent, dans des cas comme le cas colombien, ce que nous pourrions appeler une modernisation forcée, incomplète et excluante; aussi appelée modernisation négative ou modernisation armée ${ }^{14}$.

\section{Bogotá : une forêt tout en ciment}

Bogotá, la capitale de la Colombie, aborde le XXI siècle avec plus de sept millions d'habitants, soit environ $15 \%$ de la population totale du pays. En tant que district capital, son territoire n'a pas toujours été le même. En effet, celui-ci s'est étendu pour intégrer peu à peu des municipalités de la périphérie, comme en 1954 quand la ville a annexé six municipalités ${ }^{15}$ qui ont formé le District Spécial.

Fondée le 6 août 1538, la ville est devenue le centre politique et administratif du pays. Au début du $\mathrm{XX}^{\mathrm{e}}$ siècle, elle comptait une population de 100000 habitants ${ }^{16}$ et dans la première moitié du siècle elle sut maintenir un rythme de croissance inférieur à celui de villes telles que Medellín, Cali ou Barranquilla, qui ensemble constituent ce que Gouëset (1998) a appelé la « quadricéphalie ${ }^{17}$ » colombienne. Vers le milieu du $\mathrm{XX}^{\mathrm{e}}$ siècle, la suprématie urbaine de Bogotá s'affirma ${ }^{18}$, tendance qui s'est maintenue malgré le peu de distance qui la sépare des autres villes ${ }^{19}$. Actuellement, elle abrite un peu plus du cinquième de la population urbaine totale du pays, ce qui confirme sa tendance à la concentration de la population et la différencie d'autres capitales latino-américaines telles que Mexico ou São Paulo.

Il faut rappeler que tout en étant toujours conforme à une tendance à la baisse, dans les cinq dernières années Bogotá a accueilli entre un tiers et un cinquième de la population déplacée de tout le pays. Entre $80 \%$ et $95 \%$ des familles arrivant à Bogotá ont des liens avec le milieu rural, ceci est le reflet d'un changement énorme survenu en termes d'espace habité par la grande majorité des personnes déplacées. Plus la fracture territoriale est grande, plus le «bannissement s'intensifie et plus les processus de reterritorialisation deviennent lents et incertains.

La capitale colombienne apparaît comme un lieu offrant de plus grandes possibilités de survie. Elle se caractérise par une moindre capacité d'action des acteurs armés et par une plus grande concentration des ressources que peut offrir l'État au niveau de soutien. La plus grande densité de population offre la protection de l'anonymat et sa dynamique économique rend possible la rencontre d'espaces de travail qui permettent de tout recommencer à zéro. De même que dans les migrations économiques, la participation de Bogotá comme terre d'arrivée a décru, d'autres villes capitales et chefs lieux régionaux tendant à prendre le relais.

L'arrivée dans une ville inconnue pour la grande majorité des gens, signifie une rupture territoriale importante. Mais, quels sont les critères qui pèsent lors du choix de Bogotá comme destination d'exil? Le choix d'une destination est marqué tant par les décisions des autres que par les réseaux d'amitié et de parenté qui facilitent l'intégration. Parents et entourage qui offrent une aide au cœur d'une ville inconnue, pour commencer, sont des raisons suffisantes. Pour Pedro, il est clair qu' " on vient à Bogotá, le plus loin, pour être un peu plus sûrs. Ce n'est pas pour l'argent, ni parce qu'ici, comme disent certains, il y a du travail. C'est pour la sécurité. C'est plus éloigné de tous ces problèmes, plus éloigné de la mort. » 
Mais la perception de la sécurité qu'offre la ville est relative pour d'autres comme Sandra. «Ici, on n'est pas en sécurité, mais on ressent comme un peu plus de tranquillité. Là-bas, la peur, c'était de rester chez soi et qu'ils en finissent avec toute la famille ». Pour Emilia, cela a été une frustration : " en ce moment même, on pense que arrivée à la capitale, on va obtenir plus d'aide, de travail, les choses plus faciles mais ici, tout est difficile. Je connaissais déjà Bogotá mais cela fait longtemps. Quand j'avais 15 ans, je suis venue travailler ici un an, mais je ne m'y suis pas plue et je suis retournée d'où je venais ».

La vie de quartier peut amener des retrouvailles avec la violence ou encore d'autres formes de violence, de la part d'autres acteurs ou peut-être des mêmes. D'une part, la délinquance commune entretien un climat d'insécurité et impose des relations différentes avec le logement, qui doit être maintenu fermé tout le temps. D'autre part, la violence à orientation politique reste très présente. C'est le cas des assassinats sélectifs, particulièrement des assassinats de jeunes $^{20}$, perpétrés par le commando paramilitaire Bloque $\mathrm{Ca}$ pital (Bloc capital), qui bénéficie du soutien des habitants, particulièrement des commerçants locaux ${ }^{21}$. Les groupes font du porte à porte pour offrir des services de surveillance et les gens qui acceptent doivent payer une cotisation mensuelle $^{22}$. En dehors de la fonction d'ordre et de sécurité qu'ils s'arrogent en tant que milice privée, para-étatique, les paramilitaires cherchent à éradiquer toute relation existante entre les groupes guérilleros et les milices populaires. Ainsi voit-on la ville devenir le domaine privilégié des paramilitaires pour reproduire le modèle de dispute territoriale existant dans les zones rurales marginales qui désormais s'étendent aux zones de la frontière rurale-urbaine marquées par la misère.

Le nouvel espace confronte les déplacés à la précarité extrême mais aussi àl'invention de mécanismes mis en place par les femmes pour aller chercher fruits et légumes à la centrale d'approvisionnement. Virgelina explique ce qu'est cette activité :. " Je vais à Abastos récupérer de la nourriture. On nous y traite très mal. On nous dit : "Va travailler!" J'y vais tous les huit jours avec une autre voisine. On part à cinq heures du matin. Il y a plein de choses jetées. Parfois, on les ramasse et ils vous les reprennent. Parfois, on nous offre des fruits. Il faut avoir de la patience. On récupère oignons, tomates, pommes de terre, légumes, fruits, bananes ».

Des stratégies de survie culturelle permettent aussi d'assurer une continuité à ces pratiques sociales de rencontre, de fête, de célébration et de jeu. Comme dit Delgado, « entretenir des conduites culturelles singularisées a été essentiel pour que les immigrants parviennent à affronter les cadres d'exploitation et de marginalisation qu'ils ont eu si souvent à endurer. Ainsi les mécanismes de reconnaissance mutuelle entre les immigrants d'une même provenance leur a toujours donné la possibilité d'activer un réseau d'entraide et de solidarité très utile ${ }^{23} »$. Dans le cadre d'une dynamique de continuité et de fragmentation des pratiques culturelles des desplazados afro-colombiens à Bogotá, on trouve diverses attitudes et différentes conduites. Carmen, par exemple, a maintenu certaines coutumes : " J'ai quelques amis et quelques personnes que je connais, que j'ai connu ici, d'autres endroits. On joue au bingo et aux dominos, comme là-bas. Spécialement les femmes. Les hommes, pendant la semaine, quand ils n'ont rien à faire, se réunissent et jouent au billard ou aux cartes. Et le weekend, ils vont boire, parler entre hommes. » Profitant des possibilités, certains cherchent à recréer leur milieu rural dans le quartier comme c'est le cas pour Pedro et Julieta : «Ici, nous avons quelques lapins, des poules, un couple de canards et même une chèvre. " Les restrictions en termes d'espaces sont grandes; par conséquent, cette pratique n'est possible que lorsqu'on est propriétaire d'un lot.

Le passage de rural à urbain marque des ruptures au niveau du savoir-faire. Ainsi, une partie de l'offre institutionnelle est destinée à la formation. Une partie de celle-ci vise la réorientation professionnelle par le biais de cours pour élaborer des projets productifs, qui comprennent des matières telles que l'agriculture en climat froid, la comptabilité, le maniement de l'ordinateur, la gestion de projet, la création d'entreprise. D'autres cours visent la formation sociale, tels que les ateliers pour les parents ou pour les premiers secours. "J'aime bien assister aux ateliers. Ici, c'est super parce que j'ai eu la possibilité d'apprendre toutes ces choses, tandis que là-bas, on n'avait pas cette possibilité. »

La différence d'environnement rend plus profonde la nostalgie de ce que l'on a laissé. Le mythe d'Ulysse, à savoir le rêve d'un retour vers un royaume d'Itaque magnifié, ${ }^{24}$ est très fort et trouve son reflet tant dans la comparaison quotidienne de coutumes, de paysages et de jouissances, que dans les possibles articulations entre l'espace actuel et l'espace antérieur, entre Bogotá et quelque endroit rural. Le quotidien de la survie remet en mémoire avec d'autant plus de douleur et un regard idéalisé le patrimoine qu'ils possédaient et dont ils ne jouissent plus. Leurs fermes, leurs villages sont reconnus comme un territoire prodigue en ressources. Pedro insiste sur le contraste: «Regarde la situation que nous vivons. Sur nos terres, on va à la sementera [plantation], on cueille les bananes, on va avec son chien, et on chasse un animal. On revient à la maison, on vend les bananes et on vend la viande de l'animal. Ici, il faut payer le billet de transport. Là-bas on n'a rien à payer sous prétexte qu'on va d'un endroit à un autre en marchant ». 
La dépendance monétaire propre à la ville fait contraste avec le territoire qu'on a quitté où il y avait toujours du travail sur les terres, et toujours de la nourriture. La ville sous-entend un emploi, rare et mal payé, ne pouvant pas garantir la survie familiale. La rupture des espaces reproductifs et productifs dans la ville affecte tout spécialement les femmes. "Là-bas, je cherchais de l'or, dans les fermes, je coupais des bananes. On est habitués à travailler. Mais ici, c'est très difficile de faire garder ses enfants. Là-bas, on les avait avec nous, à la ferme », explique Emilia.

Presque tous possédaient une maison - ou un rancho comme ils l'appellent - où vivre avec leur famille. Cependant, ici, le travail initial, c'est trouver où vivre. On regrette non seulement le paysage, le climat et les coutumes différentes, mais aussi la carence et la misère présentes qui sont mises en face à la relative abondance et diversité dont ils disposaient dans leurs régions. Ses logements ruraux, humbles mais spacieux et adaptés sont devenus désormais des chambres louées, très petites, pas assez grandes pour permettre aux enfants de jouer. Les travaux domestiques, centrés pour les femmes sur la préparation de la nourriture et le lavage du linge, dans des contextes urbains marginaux où l'eau est rare, deviennent beaucoup plus durs. "On allait savonner, mais c'était plus pour se baigner et on revenait dans l'après midi. Là-bas, c'était amusant d'aller laver. On ouvrait ce linge sur la plage et c'était en un clin d'œil que cela séchait. Mais ici, avec ce froid et quand il pleut! Ici, l'eau nous arrive par des tuyaux et on a un lavoir » commente Julieta.

"Là-bas, il y a une forêt toute en montagnes et ici, une forêt toute en ciment. » Cette expression est le reflet de la perception d'une ville insensible avec l'autre, avec l'étranger pauvre. La douleur propre à l'abandon de « la terre » est permanente et s'intensifie quand les carences quotidiennes se chargent de rendre présentes les absences. «On s'ennuie de sa terre. Ce que l'on voudrait, c'est retourner à notre terre s'il y avait la paix, mais cela, personne ne le garantit. On a deux frères ici. Parfois, on boit un coup et les larmes commencent à couler. » Cependant, « à l'allure où vont les choses, c'est mieux que nous restions ici. Quand on aura vu qu'il y a de la tranquillité, on pourrait revenir » raconte Simón.

Vivre à Bogotá, cependant, n'est pas une décision définitive. Pour l'instant, l'attente d'un retour possible, bien que pas immédiat, est vivante. Accéder à un logement propre est le rêve entretenu par tous, un rêve sur lequel on parie comme but pour refaire son projet familial. Il symbolise l'intégration, l'insertion dans une société locale et devient même une espèce de mythe attendu de rupture avec sa condition de déplacé, d'étranger de passage.

\section{Desplazado : une catégorie qui tend à devenir substantielle et stigmatisée}

Le déplacement devient une mesure préventive quand «les choses commencent à tourner mal », ou une action imposée par les groupes armés sous la menace de "nettoyer la région ", ou bien encore il est justifié par les armées qui annoncent «on va s'envoyer des pruneaux et vous êtes en danger ». Néanmoins, concrètement, ces décisions ont différentes nuances en termes de temps, de positionnement devant les armées, d'endroits où aller, etc. En tout cas, une recomposition du territoire en fonction de la guerre va s'engendrer. Si la population demeure, non seulement les alliances vont se modifier avec les nouveaux acteurs armés qui ont le pouvoir mais encore, bien des règles de la vie en société, familiale et personnelle vont être bouleversées.

La guerre engendre en permanence des marqueurs identitaires aussi bien individuels que collectifs. Les qualifications du genre ami-ennemi, coupable-innocent, victime-bourreau, déplacé de $\mathrm{X}$ endroit, déplacé par $\mathrm{X}$ acteur, vont configurer de nouvelles identités, qui ne correspondent pas seulement à des comportements et à des actes récents mais également à leur trajectoire historique. De cette catégorisation va dépendre, dans de nombreux cas, littéralement la vie ou la mort, surtout dans des espaces locaux où les rapports sont plus directs et un contrôle minutieux des habitants peut être exercé. En fonction de ces identités assignées, les groupes de pouvoir, armés et non armés, déterminent, au nom du bien commun, la vie de nombreux habitants ruraux. Les actions et interactions entre groupes armés et non armés engendrent des repères identitaires au-delà du simple jeu de reflets ou de réponses plus ou moins mécaniques aux attributions identitaires faites par d'autres. Par conséquent, si l'un des acteurs veut changer l'identité qui lui est assignée, cela veut dire qu'il veut modifier le rapport entre les deux, parce que ce qui est en jeu, ce n'est pas seulement l'identité de l'un ou de l'autre, mais la situation qui contient le rapport ${ }^{25}$. Ainsi, par exemple, le changement d'armée dans un territoire que l'on se dispute entraîne une forte crainte des populations, car elles se savent considérées comme dangereuses par le groupe armé opposant. Ignorant comment interagir avec les autres, angoissée par les avertissements et les menaces, ne pouvant communiquer et s'informer qu'à travers les rumeurs, la population vit une situation insoutenable, à tel point qu'elle décide de partir, en quête d'un peu de sécurité et de tranquillité.

Mais les dominateurs ne sont pas les seuls à assigner des identités. Il y a aussi les autres habitants qui, alliés ou non aux premiers, qualifient ou disqualifient peu à peu voisins et personnes, connues ou non. On insiste sur le fait que "si on ne se mêle pas des affaires des autres et qu'on ne fait rien 
de mal, on vous laisse tranquille » ou l'équivalent, « il doit bien y avoir une raison si.... » Cette croyance se maintient bien qu'ils aient été eux-mêmes victimes sans avoir rien fait de mal. Les acteurs armés se voient ainsi légitimés car on responsabilise la victime, elle est responsable de sa situation. C'est ainsi que l'on intériorise des mécanismes d'impunité, d'indifférence et de consolidation de la justice privée.

La réalité du déplacement est née avec la guerre et doit renaître en plein milieu de celle-ci. Des situations et des points de vue semblables s'agencent en référents communs, et se construisent à partir de la perte, de la souffrance et de leur condition de victimes ${ }^{26}$. Il est indispensable de commencer un processus de construction du territoire, en occupant les espaces sociaux et physiques d'autres habitants et en entrant en concurrence pour de rares ressources, dans une articulation forcée et conflictuelle entre le rural et l'urbain. Le desplazado est vu également comme un transmetteur de violence. La perception que l'on a de la guerre dans cet autre pays, le pays rural, nourri par des moyens de communication de masse, où chaque groupe armé extermine l'ami de son ennemi, nous pousse à conclure que, dans tous les cas, le desplazado est l'ami, le militant ou le complice d'un groupe armé. Sa fuite est vue comme une preuve de sa culpabilité, qu'il traîne à sa suite où qu'il aille, parce que des factions des groupes armés peuvent le suivre jusque dans la ville. La transmission de violence qu'on impute au desplazado se trouve confirmée et augmentée lorsque l'on connaît ou que l'on présume quelle est leur provenance; le Caguán, l'Urabá, Córdoba, Barrancabermeja et bien d'autres endroits ont dans l'imaginaire collectif une adhésion territoriale avec les groupes armés, que l'on fait endosser à l'habitant.

Dans ce contexte de misère et d'urgence, où il faut résoudre des besoins de premier ordre, d'autres stigmates s'ajoutent à ceux qui viennent de la guerre elle-même. Comme on l'assimile aux dépossédés, le desplazado est également vu comme un délinquant potentiel : les niveaux de misère urbaine et la corrélation quasi automatique entre pauvre et délinquant, aboutit à la construction du stéréotype du desplazado comme une nouvelle recrue de la pauvreté, qui habite dans les ceintures de misère de la ville, d'où sortent, justement, les délinquants. Les desplazados contribuent ainsi à augmenter "la poudrière sociale " ou "la bombe à retardement » qui bouillonne jour après jour dans la marginalité urbaine. D'après un document du Red de Solidaridad Social (Réseau de solidarité sociale), c'est « un nouveau facteur de déstabilisation politique et économique de notre région. » Les desplazados sont, dans la sphère locale, les nouveaux demandeurs de ressources étatiques, qui rentrent en compétition avec les "pauvres historiques", anciens immigrants qui attendent une solution depuis naguère. Fonctionnaires, habitants et organisations sociales partagent souvent cette vision qui place les plus vieux demandeurs en position prioritaire. À cela vient s'ajouter la représentation de l'imposteur, qui se fait passer pour un desplazado pour profiter de la solidarité économique. On peut même être nommé « desplazado professionnel ». La méfiance freine la solidarité et impose une série d'" attestations " pour montrer aux « autres » qu'effectivement, on appartient à cette catégorie sociale. Le desplazado fait figure de nouveau mendiant dans les rues des villes, qui pour se différencier des mendiants traditionnels résume en une phrase son statut d'" être déplacé par la violence».

Depuis la guerre et la misère on construit toute une série de stigmates à travers lesquels est reconnu, évité et exclu celui qui vit dans le déplacement forcé. Cela constitue une barrière difficile à visualiser et à manipuler qui, néanmoins, traverse les nouvelles dynamiques de sociabilité dans les nouveaux contextes urbains où on cherche à reconstruire un projet de vie. Les desplazados se meuvent entre l'illusion que donne la recherche d'une reconnaissance administrative du déplacement et le poids des multiples stigmates.

\section{Solidarités, organisations et résistances urbaines}

La ville offre de plus grands répertoires de confrontation au niveau des actions de fait et des discours exprès qui mettent l'État face à ses responsabilités en ce qui concerne le desplazamiento et exigent de lui le respect des droits de déplacés, quitte à faire appel aux instances légales. Cependant, tous les déplacés de Bogotá ne font pas pour autant appel à ces répertoires, car une espèce d'autocensure sévit, motivée par la peur, l'obéissance à l'autorité et la perspective stratégique de créer plus d'alliances que de conflits. Ainsi, l'accès à des espaces d'articulation, bien que passagers et naissants, s'en est trouvé favorisé entre organisations de déplacés. En ce processus là, la dynamique du secteur tertiaire, donc des ONG, est une des plus actives du pays et représente une opportunité politique importante et diverse pour les organisations de déplacés. L'accès aux évènements, à la formation, aux discours institutionnels, etc. permet aux groupes présents à Bogotá d'enrichir leur propre discours, discours exprimant leurs besoins, d'un discours sur leur droit à une réponse de la part de l'État.

Fédérer tous les efforts pour résoudre les problèmes quotidiens est une possibilité surgie de la nouvelle situation de dépouillement qu'a laissé le desplazamiento forcé. Bien que de nombreuses actions communes n'aient pas de caractère formel, elles contribuent cependant à refaire les relations sociales dans de nouveaux contextes, à partir du référent identitaire contradictoire de «ser y estar despla- 
$z a d o »$ (déplacé permanent et temporaire). On retrouve ainsi des solidarités nées à partir d'initiatives des desplazados eux-mêmes, en tant qu'initiatives dont les institutions font la promotion et auxquelles elles apportent leur soutien. Ceci s'illustre par exemple par les soupes populaires conçues par les femmes, qui peuvent évoluer vers des actions de portée et de durabilité plus grande. D'autres formes d'action conjointe, même si elles ne sont pas organisées, consistent en la récupération des déchets des grandes industries agricoles ou l'entraide entre voisins pour garder les enfants quand les parents vont travailler ou pour s'occuper des malades. Même si ces stratégies de solidarité ponctuelle entre personnes pauvres peuvent être vues comme quelque chose de faible portée, elles constituent une force importante tant dans la survie des familles que dans la constitution de nouveaux réseaux sociaux qui peuvent avancer vers des processus à plus longue échéance.

La nécessité de canaliser les ressources et l'attention institutionnelle, ainsi que les exigences de la part de ces mêmes institutions d'aide, a généré une croissance rapide du nombre d'organisations de desplazados qui, jusqu'en 1997, étaient très rares. En suivant un schéma traditionnel et généralisé pour l'action collective, les personnes desplazadas se regroupent en associations et en collectifs, lesquels se constituent fréquemment à partir de l'hétérogénéité et de la conjoncture de la rencontre, plus qu'à partir de l'homogénéité des lieux d'origine. La plupart du temps, ce sont des organisations qui ont une existence légale et qui sont enregistrées auprès de l'État, démarche qui devient une condition pour les relations avec celui-ci.

Réplique des Juntes d'Action Communales (JAC), modèle le plus étendu dans le pays tant au niveau urbain que rural, ces organisations représentent les intérêts d'un secteur de la population auprès du gouvernement local, régional et national en vue d'assurer la gestion et d'investir des ressources. Depuis le schéma technocratique qui s'est imposé avec la décentralisation et la modernisation de l'État, les demandes des colons passent par « l'élaboration de projets ». Ces derniers sont une formalité dans l'intermédiation institutionnelle avec l'État et les ONG. Cette logique suppose des niveaux éducatifs et de gestion de l'information dont, dans la pratique, on ne dispose pas et qui ne correspondent pas avec les urgences et les situations limites du desplazamiento forcé. Cette formalité finit par être satisfaite en faisant appel à des intermédiaires qui élaborent le projet. Cependant, la différence entre les temps bureaucratiques et ceux des besoins et des rythmes des groupes permettent difficilement des processus satisfaisants de planification participative. Dans de nombreux cas, il n'y a aucune correspondance entre ces efforts et les processus de sélection technique et opportune de réponses, car les mécanismes traditionnels du clientélisme et de la corruption continuent à primer sur les organismes gouvernementaux. D'autre part, l'aide reçue de l'État et des organismes d'aide subit fréquemment une forte ingérence qui conditionne les formes organisationnelles ainsi que les logiques et les dynamiques des groupes ${ }^{27}$.

Les difficultés, dans ces processus collectifs, sont diverses. Les énergies des dirigeants sont concentrées sur le succès de la reconnaissance légale et sur de multiples démarches pour concrétiser et gérer les demandes de soutien de la part de l'État. Les familles associées font quelques apports en argent pour ces démarches et participent à des réunions périodiques, mais habituellement la résidence dispersée rend difficile la rencontre et la communication permanente. En même temps, la gestion quotidienne de la subsistance génère une concurrence entre les besoins familiaux et le temps dont a besoin l'organisation ${ }^{28}$. Maintenir la dynamique et la force de l'organisation dépend, dans une large mesure, de sa capacité à faire la preuve de succès matériels vers des solutions stables. Les délais et l'absence de réponses affaiblissent les motivations des leaders et des associés. Les associations et autres groupements de desplazados reproduisent fréquemment la corruption, l'autoritarisme et la manipulation, ce qui restreint les possibilités de recréer des expériences alternatives d'organisation sociale. La modalité de représentation qui délègue à quelques-uns uns le pouvoir de la parole délivre aussi la responsabilité des actions en diminuant la participation des associés.

Il est nécessaire de souligner que le processus menant à redessiner des réseaux sociaux en ville est plus facile quand on dispose d'un référent identitaire qui existe déjà, un sens du « nous » qui contribue à une mobilisation à travers l'action collective. Ceci permet en effet la construction de consensus internes autour du leadership et des normes, beaucoup plus fluides et présentant beaucoup plus d'éléments de confiance du fait qu'il y a déjà une base de mémoire partagée qui sert de " niche morale » à la sociabilité institutionnelle de l'action collective. L'inexistence préalable de ce référent, comme c'est le cas pour bonne partie des paysans déplacés en ville, n'empêche pas cependant des actions collectives de surgir. Mais le rythme de construction des consensus et des relations de confiance va, dans ce cas, être beaucoup plus lent, beaucoup plus ambigu et sélectif.

On peut dire que la mobilisation des opportunités politiques offertes par Bogotá aux déplacés dépend de trois types de dynamiques : d'abord, l'articulation et le positionnement dans la chaîne des intermédiaires institutionnels qui, bien qu'ils apportent leur soutien, conditionnent aussi cette mobilisation; ensuite, les rythmes, processus et consensus internes à chaque groupe pour assumer les risques potentiels que la mobilisation implique; et enfin, la décision 
de chacun, en tant qu'individu, de participer ou non à la poursuite d'actions collectives, en fonction de ses propres intérêts, de ses propres besoins et expériences.

Les actions collectives, organisées ou pas, orientent aussi les énergies vers la protestation contre l'État. La pression s'est imposée dans la mesure où les réformes promises ne sont pas engagées. Les paysans, convertis désormais en citadins par la force de la guerre, cherchent à s'exprimer et demandent des actions effectives qui viennent résoudre ou compenser, ne serait-ce que partiellement, leur situation de misère et de manque de protection.

Une caractéristique de ces manifestations est leur développement dans les espaces urbains et tout spécialement dans les capitales. Ceci correspond à l'inertie d'une néocentralisation qui continue à concentrer dans les cités les décisions et les ressources. Suivant une tendance historique, les paysans vont à la ville chercher la solution à leurs problèmes parce que c'est là qu'ils trouvent la figure de l'État et non pas dans les campagnes. Dans un échantillon de protestations qui se sont succédé dans le pays entre 1997 et 2001, on a trouvé que quatre actions collectives utilisant la pression sur dix ont été réalisées à Bogotá, tant parce que c'est un point stratégique au niveau des institutions, que parce que la capitale offre de meilleures garanties de «sécurité. " Cependant, les expressions de protestation ont commencé à se manifester à l'échelle régionale, les villes capitales en étant les épicentres.

L'État est un interlocuteur reconnu, dans la mesure où légalement, il a la responsabilité d'apporter des réponses opportunes aux multiples besoins de ceux qui se trouvent desplazados. Ainsi, près de la moitié des actions menées à bien se font par le biais de "prises » d'organismes gouvernementaux. Ensuite viennent les organismes religieux qui, estiment les desplazados, vont respecter leurs droits à la protestation. La prise d'espaces publics, tels que les coliseos (stades) et les places municipales, ne correspondant par toujours à une forme de pression mais souvent à la recherche d'un espace où s'abriter. Cette forme est sous représentée, car habituellement les gouvernements locaux font appel à l'installation, sur ce type d'espace, des personnes en desplazamiento collectif. Ces espaces collectifs comprennent les écoles et les centres communautaires. Finalement, on trouve les prises d'organismes internationaux qui, sans être trop nombreuses, ont considérablement frappé l'opinion publique et contribué de manière importante à « internationaliser $»$ le problème du desplazamiento ${ }^{29}$.

Dans certains cas le gouvernement a répondu par des concertations formelles et des promesses d'attention qui, dans bonne partie des cas, n'ont pas été respectées, provoquant une dé-légitimation encore plus grande. Dans certains cas les groupes paramilitaires ont contribué àdissoudre ces actions collectives par le biais de menaces, d'assassinats ou d'ordres péremptoires sous le prétexte de résoudre des situations qui incommodent le reste de la population $^{30}$.

L'exercice revendicatif récent de la part des personnes desplazadas, mouvement encore naissant et un brin tardif si l'on tient compte de l'existence du phénomène durant cette décennie, est fortement marqué par la peur des représailles. Selon les termes de Tarrow, ces mouvements pourraient être identifiés comme des " actions collectives contentieuses ", dans la mesure où elles émanent de personnes dépourvues d'accès régulier aux institutions, qui agissent au nom de revendications récentes et qui sont vues comme une menace. Ces actions émergentes qui constituent un défi collectif pourraient bientôt constituer les prémices d'un mouvement social ${ }^{31}$.

\section{Conclusion}

Développement économique, ressources institutionnelles et une moins grande vulnérabilité face à la guerre constituent trois revendications que l'on retrouve au sein des flux de populations déplacées vers Bogotá par la violence. La primauté urbaine de Bogotá vue depuis les régions et depuis les conditions d'intensification du conflit armé dans le pays emmène à renforcer l'imaginaire de la métropole comme un lieu qui offre de plus grandes promesses de sécurité. Suivant les flux traditionnels des migrations économiques, les itinéraires du desplazamiento forcé se sont orientés en bonne partie vers la capitale du pays, suivant des rythmes beaucoup plus intenses.

Bien qu'ils arrivent dans une ville où ils rencontrent des manifestations d'un développement économique, avec ses avenues, ses grands édifices, ses centres commerciaux et ses usines, les nouveaux venus vont être situés dans les quartiers des pauvres. Depuis la ségrégation urbaine, ils alimentent l'espoir de lendemains meilleurs à partir du reflet de ce que possèdent les autres et à partir des choses dont les autres profitent. Coincés dans un quotidien misérable, les desplazados vivent à la fois la dureté et l'illusion du bien-être que peut offrir la ville. Fuyant les acteurs armés et la guerre dans les campagnes, ils se retrouvent dans la ville, face à l'extension de cette guerre et face à d'autres violences qu'ils doivent affronter. La guerre avance vers les villes et la violence sociale y a des manifestations intenses. Le mythe de l'endroit sûr aussi se dissipe.

La rencontre avec d'autres déplacés dans les mêmes circonstances que soi permet de tisser de nouvelles sociabilités improvisées, fragmentées et ambiguës. Se regrouper pour se montrer publiquement comme acteurs collectifs, voilà une possibilité qui devient prérequis et nécessité face à l'indifférence, à l'inefficacité et à la suspicion. La relation 
avec les organismes gouvernementaux et les ONG, entités éloignées des déplacés, exige une série de légitimités, de connaissances, de démarches et d'efforts qui requièrent la formation d'organisations avec des porte-parole. La parole faite appel à une plus grande force quand elle est soutenue par un collectif, même si une telle délégation dépouille tous les associés de leur propre parole.

Cependant leurs vécus personnels de manque de protection dans leurs lieux d'origine les emmène fréquemment à vouloir rester ici, nourris par l'espoir de pouvoir résister mais aussi par le désespoir de n'avoir pas d'endroit vers où partir. Les possibilités de gérer et de gagner quelques ressources et quelques réponses à leur situation avec les organismes qui contribuent aussi à les fixer en ville. Malgré les promesses non tenues, les expériences ratées et l'incertitude des innombrables démarches, la persistance à croire que «quelque chose en sortira »'impose. En somme, la permanence en ville devient un mirage du lieu d'accueil. Malgré la preuve du contraire, on soutient l'attente d'un futur meilleur, à défaut d'autre horizon et au milieu de la lassitude provoquée par l'instabilité et l'incertitude.

\section{Notes}

1. Extrait du texte de l'oeuvre fournie par l'ONG Taller de Vida (Juin 2001).

2. La promulgation de la loi 387 de 1977 a créé la catégorie juridique de desplazado et également précisé la responsabilité de protection que l'État doit leur donner.

3. La recherche s'est fait entre 1996 et 2001.

4. Voir Congreso Nacional de Paz y País (Congrès National pour la Paix et le Pays), «Relatoría Comisión : Conflicto Agrario, Seguridad Alimentaria y Cultivos de Uso Ilícito » (Bogotá, du 9 au 11 mai 2002).

5. Les promesses de la modernité se concrétisent à travers une citoyenneté abstraite, la régulation et le traitement des conflits par l'État, le contrôle de la science, le progrès économique et une distribution plus large de ses "bénéfices ». M. Palacios, "Conflicto y modernidad en Colombia ", Documentos (Santafé de Bogotá : Banco de la República, 1992), 13.

6. Au niveau international, en Afrique, par exemple, les routes du desplazamiento forcé vont des villes jusqu'aux camps de refugiés et desplazados. D'autres espaces sont des endroits difficiles d'accès, comme les bois. M. Lavergne, « De la cuvette du haut-Nil aux faubourgs de Khartoum. Les déplacés du Sud-Soudan ", dans Déplacés et Réfugiés. La mobilité sous contrainte, sous la dir. de V. Lasailly-Jacob, J.-Y. Marchal et A. Quesnel (Paris : IRD Éditions, 1999).

7. Le massacre de Segovia (Antioquia), par exemple, une ville d'environ 50000 habitants, où ont été assassinées presque 40 personnes en un seul après-midi, met en évidence l'impotence d'une collectivité face au pouvoir des armes, surtout quand l'attaque s'est fait en alliance avec quelques autorités locales.

8. On aborde ce sujet dans la troisième section du présent article.
9. Être voisin du bâtiment de la police peut être vu comme un danger que la population voudrait éviter.

10. L'alliance entre paramilitaires et armée est fréquente. Voir, par exemple, des cas sur lesquels ont fait enquête diverses instances d'État et ONG, comme les massacres de Naya, Carmen de Bolívar et Barrancabermeja.

11. Ce type de manifestation est habituellement signalé comme une action dirigée par la guérilla et soutenue par des « forces obscures ». En 1998 ont été enregistrées à Bogotá 12 tomas (occupations) de bureaux gouvernementaux et d'endroits publics, d'une durée d'entre un jour et trois mois, par des organisations de desplazados qui voient de telles manifestations comme une stratégie de pression qui vise des solutions rapides. À Barrancabermeja 10000 paysans sont restés 45 jours. Voir Groupe de soutien aux desplazados, «Informe sobre desplazamiento Interno Forzado en Colombia en 1998 » (Santafé de Bogotá : Documente, 1998).

12. A. Reyes, "Conflicto y territorio en Colombia ", dans El campesinado en Colombia hoy; diagnóstico y perspectivas (Santafé de Bogotá : ECOE, Universidad Javeriana, 1991).

13. Le processus d'urbanisation s'est nourri fondamentalement du transfert net de population entre zones rurales et zones urbaines. L'urbanisation est considérée comme un processus fini qui s'épuise quand l'augmentation de la proportion de la population totale résidant dans des localités urbaines est entravée, c'est-à-dire que toute la population devient urbaine et que la composante rurale est en voie d'extinction. M. Villa, «Distribución Espacial y Migración de la población en América Latina ", dans Migración, Integración regional y transformación productiva, sous la dir. de D. E. Celton (Córdoba : Universidad Nacional de Córdoba, 1995).

14. Voir, par exemple, F. Gonzalez; T. Vasquez et I. Bolivar, Violencia Política en Colombia (Bogotá : CINEP, 2003).

15. Il s'agit des municipalités suivantes : Usme, Fontibón, Usaquén, Bosa, Engativá et Suba.

16. En tant que seconde ville la plus peuplée de Colombie, Medellín comptait 53936 habitants. V. Gouëset, Bogotá: nacimiento de una metrópoli. La originalidad del proceso de concentración urbana en Colombia en el siglo XX (Bogotá : Tercer Mundo Editores, Observatorio de Cultura urbana, CENAC, IFEA, FEDEVIVIENDA, 1998).

17. Ibid.

18. Selon Gouëset, la suprématie urbaine est un concept rapproché de la concentration rapide en pôle urbain qui conjugue exode rural massif, explosion démographique et industrialisation. Ce phénomène, original dans le contexte latino-américain, est plutôt récent en Colombie et serait déphasé de presque un demi-siècle avec ce qui s'est passé dans d'autres pays du continent. Ibid.

19. Bogotá fait deux fois Medellín et figure parmi les quatre villes qui recueillent près de $30 \%$ de la population nationale.

20. La surveillance est assurée par des jeunes qui s'envoient des signaux à travers des sifflets. Ces groupes assurent un travail de «limpieza social », assassinant les gens, particulièrement de jeunes hommes, signalés comme subversifs, délinquants, 
drogués ou n'importe quelle étiquette considérée comme dangereuse. Témoignage d'une jeune non déplacée habitante du secteur de Ciudad Bolívar, mai 2001.

21. Voir, par exemple, "Andanzas de las AUC en Bogotá ", El Espectador, 10 Juin 2001.

22. En février 2001, elle s'élevait à 10 USD.

23. M. Delgado, Ciudad líquida, ciudad interrumpida. (Medellín: Editorial Universidad de Antioquia, 1999), 100.

24. F. A. Sayad, «Le retour, élément constitutif de la condition de l'immigré », Migrations Societé 10, nº 57 (1998) : 9-45.

25. I. Toboada-Leonetti, «Stratégies identitaires et minorités: le point de vu du sociologue », dans Stratégies identitaires (Paris : Presses Universitaires de France, 1997).

26. L. Boltanski, La souffrance à distance. Moral humanitaire, médias et politique (Paris : Métailié, 1993).

27. Comme les formes coopératives et la propriété de la terre en commun pour les ré-établissements ruraux de population desplazada. Voir F. Lozano et F. E. Osorio, De víctimas de la violencia a buscadores de la paz (Santafé de Bogotá : Universidad Javeriana y Acción Cultural Popular, 1999) et F. E. Osorio, «Reasentamientos rurales de población campesina desplazada ", Les Cahiers ALHIM, n 3 (2001).

28. Sur les expériences avec ces caractéristiques voir, par exemple, Rodríguez (1998), Lozano et Osorio, Mencoldes (2000).
29. Une des expériences d'action collective se réfère à l'occupation du Siège du Comité International de la Croix Rouge à Bogotá, en décembre 1999. Voir F. E. Osorio, Los desplazados. Entre survie et résistance, identités et territoires en suspens (Lille: ANRT, 2005).

30. Comme dans le cas de la prise du Coliseo de Buga, où les familles situées reçurent l'ordre des paramilitaires d'évacuer les lieux dans un délai de huit jours, délai qui a été respecté. Dans le cas des invasions en Mars 2001 à Montería, au terme de troubles avec l'Armée et la police, les paramilitaire obligèrent à évacuer à travers des menaces et plusieurs assassinats. Ainsi, ce fut eux qui offrirent une parcelle de terre proche de la ville pour y créer une espèce de quartier.

31. S. Tarrow, Poder en movimiento (Madrid : Alianza Editorial, 1997).

Flor Edilma Osorio Pérez est professeure chercheuse du Departemento de Desarrollo Rural y Regional, Facultad de Estudios Ambientales y Rurales, Universidad Javeriana, Bogotá, Colombie. 